\title{
DEVELOPING OF DECISION SUPPORT SYSTEM FOR BUDGET ALLOCATION OF AN R\&D ORGANIZATION
}

\author{
Anisha Mohan ${ }^{1}$, R Sasikumar ${ }^{2}$ \\ ${ }^{1}$ M Tech Industrial Engineering and Management, Rajiv Gandhi Institute of Technology, Kerala \\ ${ }^{2}$ Professor, Dept. of Mechanical Engineering, Rajiv Gandhi Institute of Technology, Kottayam, Kerala
}

\begin{abstract}
The problem of optimizing fund allocation in the public sector through performance based budgeting is a complex process. This paper considered only a very small part of this process, namely the final allocation of public funds through goal programming technique. While analyzing the last nine years of budget data in an $R \& D$ organization, it is inferred that there is wide gap between fund utilization and its allocation. The paper proposes a performance based model to assist decision makers in assessing the $R \& D$ programmes of an organization and accordingly make budget allocation in a more realistic and accurate manner than before. The model has been finally implemented using Excel spreadsheet and the same can be used as a DSS (Decision Support System) for the performance-based allocation of public funds. This study has made an approach to assess the influence of priority and risks associated with R\&D programmes using Fuzzy Set Theory (FST).
\end{abstract}

Keywords: Performance -based Budgeting, Goal Programming, Fuzzy Set Theory ****

\section{INTRODUCTION}

In the present scenario of economic context, optimum utilization of public funds requiring optimum public fund allocation is a dynamic and complex process. The problem of optimizing expenditure in the public sector was first discussed in early 1990s especially in the U.S.A. Various models of performance based budgeting were developed and implemented from then. Given the complexity of the operational context, public funds allocation is difficult to optimize[1].This paper addresses the problem of public fund allocation in an $R \& D$ organization using a performance based goal programming model with fuzzy logic .

Performance based budgeting has been emerged as a tool for optimizing the public funds allocation. It has been widely employed in several OECD countries. Performance budgeting focuses on results rather than inputs compared to traditional budgeting. It helps managers to have more confidence in their policy making decisions. Here we develop a decision support system to implement performance based budgeting to improve the existing fund allocation in the concerned R\&D organization.

The organization of this paper is as follows: First section provides an introduction to Performance based budgeting and the problem addressed in this paper. The second section gives a short review of the literature referred. The third section describes the problem identified. The methodology is described in detail in the fourth section. A case study is included in the fifth section.Section six summarizes the study in a brief manner.

\section{LITERATURE REVIEW}

This section deals with a brief review of researches conducted in the area of decision making in order to identify the approach and tool to be adopted in the work. The literature shows several applications of goal programming (GP) and fuzzy logic techniques. It is seen from the literature that GP has been the most widely used multiobjective technique in management science. Among the different risk analysis techniques, the application of Fuzzy Set Theory (FST) to risk analysis seems appropriate from the review of literature conducted.

\subsection{Goal Programming}

Charnes et al. developed Goal programming (GP) in 1955. GP is a multi-objective programming technique. The ethos of GP lies in the Simonan concept of satisficing of objectives[2]. Simon introduced the concept of satisficing, a word that originated in Northumbrial where it meant "to satisfy". Satisficing is a strategy for making decisions in the case that one has to choose among various alternatives which are encountered sequentially, and which are not known ahead of time [3].

GP is an important technique for decision making problems where the decision maker aims to minimize the deviation between the achievement of goals and their aspiration levels. It can be said that GP has been, and still is, the most widely used multi-objective technique in management science because of its inherent flexibility in handling decisionmaking problems with several conflicting objectives and incomplete or imprecise information $[4,5,6]$. 
Goal programming (GP) is a mathematical programming approach that incorporates various goals or objectives which cannot be reduced to a single dimension. Charnes and Cooper originated GP to solve goal-resource problems, which when modeled with linear programming techniques were found to have infeasible solutions. GP is also a good approach to solve multi-criteria decision making problems with conflicting objectives [7]. The weighted GP for PS model usually lists the unwanted deviational variables, each weighted according to their importance. Weighted Goal Programming (WGP) attaches weights according to the relative importance of each objective as perceived by the decision maker and minimizes the sum of the unwanted weighted deviations [8]. From the literature review, it is observed that Goal Programming is a powerful tool to analyze the influence of decision variables and hence the same tool is used to optimize fund allocation for the Technology Development Programmes.

\subsection{Fuzzy Logic}

A fuzzy set is a class of objects with a continuum of grades of membership. Such a set is characterized by a membership (characteristic) function which assigns to each object a grade of membership ranging between 0 and 1 . Fuzzy logic is a set of mathematical principles for knowledge representation based on degrees of membership. It deals with degrees of membership and degrees of truth. It reflects how people think and attempts to model the sense of words, decision making and common sense [9].

After Zadeh introduced the concept of fuzzy sets and theory, researchers such as Kangari and Riggs, Peak, Tah and McCaffer, Wirba, Carr and Tah, Cho, Choi , Lyons and Skitmore, Baker and Zeng, Dikmen, Zeng, Wang and Elang, Karimiazar and Nieto used fuzzy set theory (FST)based risk modeling and analytic methods that deal with illdefined, vague, imprecise, and complex risk analysis problems [10]. Ever since Zadeh's contributed in 1965 to this new field of fuzzy logic, there has been much literature in this field.

Zeng, et al. hybridized fuzzy reasoning in 2007 and the AHP approach to handle subjective assessments and prioritize diverse risk factors, respectively[11]. Karimiazari, et al. proposed in 2011 an extended version of the Technique for Order Preference by Similarity to an Ideal Solution (TOPSIS), which resolves the multi-criteria risk assessment model under a fuzzy environment [12].

It is well accepted that Fuzzy Set Theory (FST) provides a useful way to deal with ill-defined and complex problems in decision making by quantifying imprecise information, incorporating vagueness, and making decisions based on imprecise and vague data[10]. The method allows for the translation of a subjective judgment given in linguistic expressions (i.e., "low," "high," etc.) into mathematical measures. From the literature review, it is seen that Fuzzy Set Theory is used to assess the risk and priority factors in various applications.

\section{PROBLEM IDENTIFICATION}

The existing fund allocation and utilization for $R \& D$ activities in the organization is studied and a graph is plotted with the available data on R\&D Fund Allocation \& Utilisation in the last nine years. From figure 1, it is seen that there exists significant variation between the fund allocation and utilization in R\&D. In the initial years, expenditure is even less than half of the allocated funds. A huge amount is unutilized and the same is lapsed at the end of the financial year. This is mainly due to the methodology adopted by the organization. They are allocating funds every year based on certain thumb rule. Hence a scientific method is suggested in this paper to address issues relating the budgeting process.

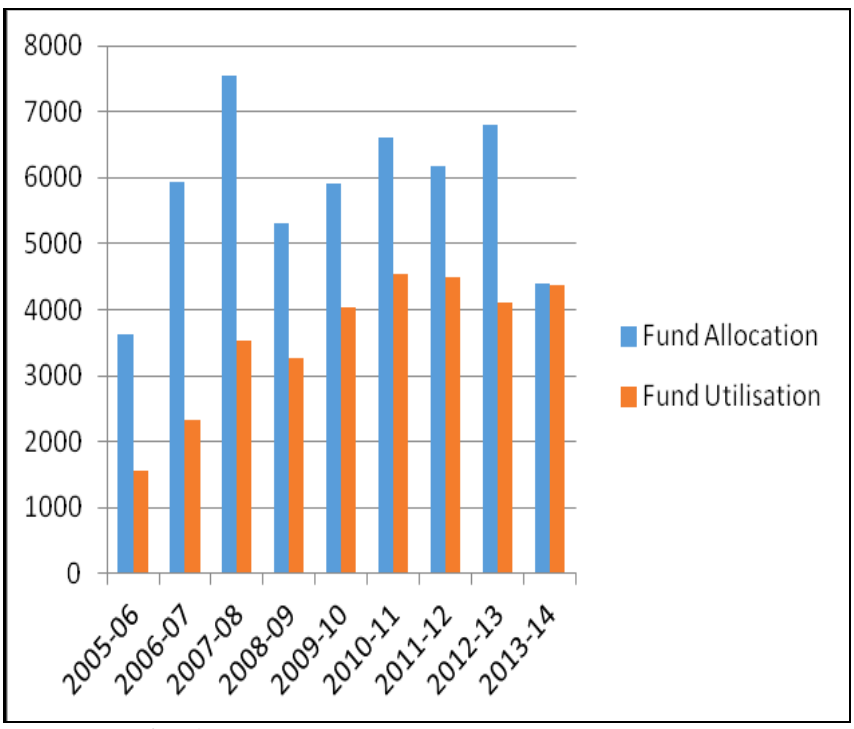

Fig-1: R\&D Fund Allocation \& Utilisation

All R\&D activities involve a Technology Life Cycle (TLC). During the initial phase of TLC, uncertainties are more. Hence the variation between allocation and utilization of funds is also more. It is also seen that improvement in expenditure during 2008-12 is due to maturing phase of technology.

\section{METHODOLOGY}

The existing fund allocation and utilisation for R\&D activities is studied and a graph is plotted with the available data on R\&D Fund Allocation \& Utilisation in the last nine years. After analysing the data, it is inferred that there exists a wide gap between the allocated and utilized funds. A performance based budgeting model is proposed in the following section based on the goal programming optimization method for improved allocation of funds. For this, mathematical modeling of fund allocation is done for formulating the objectives and constraints. The input parameters to the model are determined through fuzzy set theory. This performance based model based on goal programming is finally implemented in excel spreadsheet and the equation for the model is optimized using the excel tools. 


\subsection{Performance based Budgeting Model}

The model described here is based on goal programming optimization method. This model helps to improve the allocation in $\mathrm{R} \& \mathrm{D}$ as it allocates funds based on results rather than inputs.

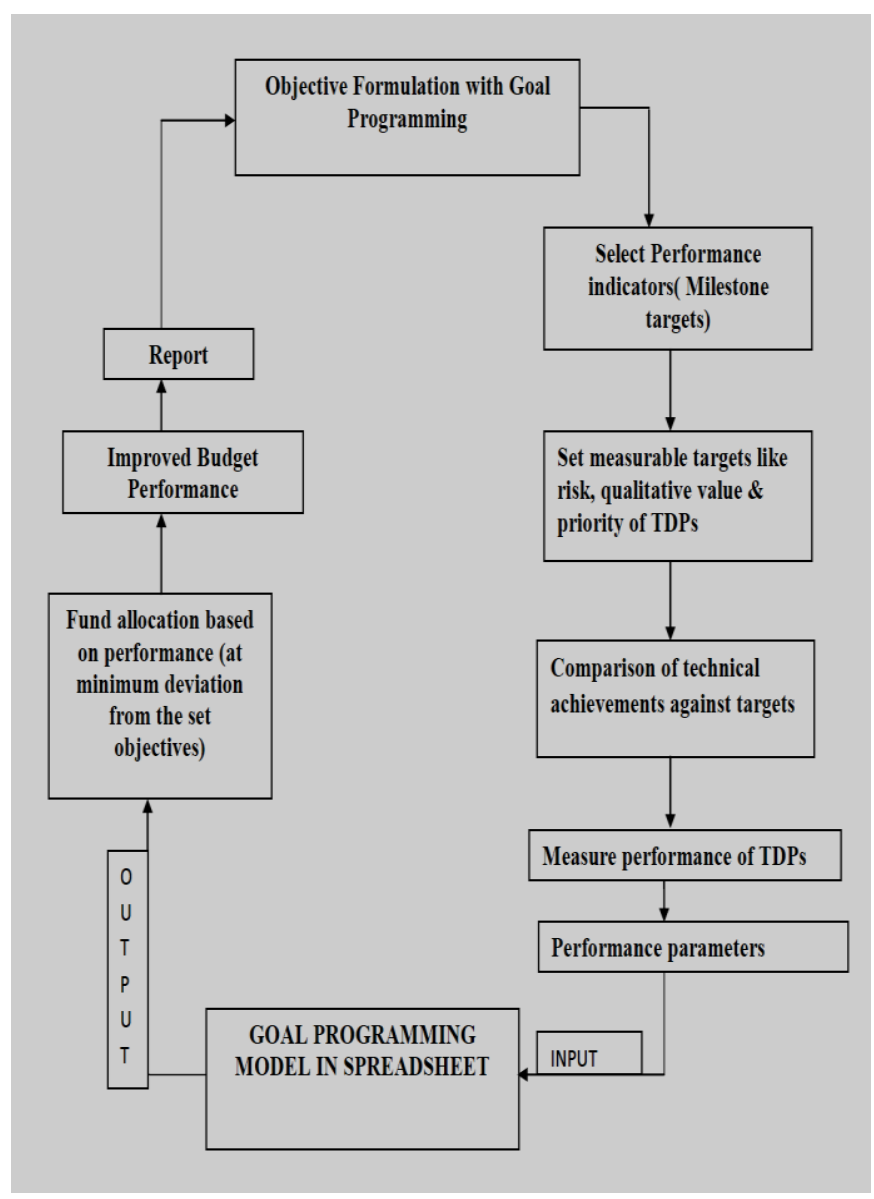

Fig-2: G P Model for Performance based budgeting

The overall procedure of the suggested goal programming model for performance based budgeting is depicted in fig. 2 . The model starts with formulating objectives through mathematical modelling which is explained in the next section. Then performance indicators like milestone targets and other input parameters like risk, priority of R\&D Programmes are selected. These parameters with higher weightage to performance indicator are given as input to goal programming model. The model outputs fund allocation based on performance and thereby improves budget performance.

\subsection{Mathematical Modelling}

The mathematical model developed in this section based on goal programming optimization method illustrates how to establish, based on some performance criteria, the amount of funds $\left(x_{i j}\right)$ to be allocated for a programme $i$ in $\mathrm{j}^{\text {th }}$ period. The problem of optimizing the allocation of funds is the optimal identification of amounts allocated to each programme, from a set of $\mathrm{N}$ programmes, so that we have the smallest deviation from the following objectives of the concerned organization:
- Minimize the risk of programmes selected for funding

- Maximize the performance qualitative value

- Maximize the experts' preference value

Table - 1: The variables used in the mathematical model

\begin{tabular}{|c|c|}
\hline Variable & Description \\
\hline $\mathrm{X}_{\mathrm{ij}}$ & $\begin{array}{l}\text { the amount allocate to programme } \mathrm{i} \text { in period } \\
\mathrm{j} \text {, where } \mathrm{i}=1,2, \ldots \mathrm{N} \text { and } \mathrm{j}=\mathrm{I} .2 \ldots . \mathrm{T}\end{array}$ \\
\hline $\mathrm{d}_{\mathrm{ij}}$ & $\begin{array}{l}\text { binary variable; } 1 \text { indicates that the } \\
\text { programme } \mathrm{i} \text { is funded in period } \mathrm{j} \text {, and } 0 \text { if it } \\
\text { is not funded, where } \mathrm{i}=1,2 \ldots . \mathrm{N} \text { and } \mathrm{j}=12 \text {, } \\
\ldots \mathrm{T}\end{array}$ \\
\hline $\operatorname{pmin}_{\mathrm{ij}}$ & $\begin{array}{l}\text { minimum threshold of funds allocation for } \\
\text { programme } \mathrm{i} \text { in period } \mathrm{j} \text {, where } \mathrm{i}=1,2 \ldots . \mathrm{N} \\
\text { and } \mathrm{j}=12, \ldots \mathrm{T}\end{array}$ \\
\hline $\operatorname{pmax}_{\mathrm{ij}}$ & $\begin{array}{l}\text { maximum threshold of funds allocation for } \\
\text { programme } i \text { in period } j \text {, where } i=1,2 \ldots . \mathrm{N} \\
\text { and } \mathrm{j}=12, \ldots \mathrm{T}\end{array}$ \\
\hline $\mathrm{w}_{\mathrm{o}}^{+}$ & $\begin{array}{l}\text { the weight of deviation from an objective } o \\
\text { by exceeding the acceptable threshold, where } \\
\mathrm{o}=1,2 \ldots .0\end{array}$ \\
\hline $\mathrm{w}_{\mathrm{o}}^{-}$ & $\begin{array}{l}\text { the weight of deviation from an objective } o \\
\text { by placing the obtained solution under an } \\
\text { acceptable threshold, where } 0=1,2 \ldots \ldots 0\end{array}$ \\
\hline$r_{i}$ & $\begin{array}{l}\text { the risk associated with programme } i \text {, where } \\
\mathrm{i}=1,2, . . \mathrm{N}\end{array}$ \\
\hline $\mathrm{R}$ & $\begin{array}{l}\text { the maximum tolerable risk associated with } \\
\text { all the programmes selected for funding }\end{array}$ \\
\hline $\mathrm{q}_{\mathrm{i}}$ & $\begin{array}{l}\text { the qualitative value of programme } i \text {, where } \\
\mathrm{i}=1,2, . . \mathrm{N}\end{array}$ \\
\hline $\mathrm{Q}$ & $\begin{array}{l}\text { the total amount of quality expected from } \\
\text { implementing all the programmes selected for } \\
\text { funding (the degree to which all funded } \\
\text { programmes achieve their established } \\
\text { performance indicators) }\end{array}$ \\
\hline $\mathrm{p}_{\mathrm{i}}$ & $\begin{array}{l}\text { the experts' preference for the programme } \mathrm{i} \text {, } \\
\text { where } \mathrm{i}=1,2, \ldots \mathrm{N}\end{array}$ \\
\hline $\mathrm{P}$ & $\begin{array}{l}\text { the overall experts' preference expected from } \\
\text { implementing all the programmes selected for } \\
\text { funding }\end{array}$ \\
\hline
\end{tabular}

Formally the above objectives may be described as in the following formulas:

1. Risks minimization: $\operatorname{Min}(\Sigma \Sigma$ rixijdij $)$

2. Performance maximization: $\mathbf{M a x}(\boldsymbol{\Sigma} \boldsymbol{\Sigma} \mathbf{q i x i j d i j})$

3. Experts' preference maximization: Max( $\mathbf{\Sigma}$ pixijdij)

where ri, qi, pi, xij, dij denotes the risk associated with programme $i$, the performance qualitative value of programme $i$, the experts' preference for the programme $i$, the amount allocate to programme $\mathrm{i}$ in period $\mathrm{j}$, binary variable; 1 indicates that the programme $i$ is funded in period $\mathrm{j}$, and 0 if it is not funded, where $\mathrm{i}=1,2 \ldots . \mathrm{N}$ and $\mathrm{j}=$ $12, \ldots \mathrm{T}$ (number of time periods for which the planning is realized) respectively. By introducing the auxiliary variables yo+, yo- $\geq 0$, where $o=1,2,3$ is objective's index, the problem may be reduced to minimizing the function which describes the deviations from these objectives:

$$
\operatorname{Min}\left(\left(\Sigma 3 o=1\left(w o^{+} y o^{+}+w o^{-} y o^{-}\right)\right)\right.
$$


where wo+ and wo- are the weights for exceeding the upper or falling below the lower acceptable threshold for objective 'o'. Since in our case the objectives are to maximize the quality and experts' preferences, they have only lower limits, then $\mathrm{w} 2+=\mathrm{w} 3+=0$; similarly, risk minimization has only an upper limit, then $w 1-=0$. Under these conditions we can rewrite the objective function as:

$$
\operatorname{Min}\left(w 1^{+} * y 1^{+}+w 2^{-} * y 2^{-}+w 3^{-} * y 3^{-}\right)
$$

Usually, in GP models, the objective functions have to satisfy two types of constraints: a) constraints on goals/objectives that should be entirely or partially satisfied; and b) system constraints imposed by the real economic environment and consequently become mandatory. In our case, we have the following constraints on goals/objectives:

$$
\begin{aligned}
& \Sigma \Sigma r_{i} x_{i j} d_{i j}-r^{+}=R \\
& \Sigma \Sigma q_{i} x_{i j} d_{i j}+q^{-}=Q \\
& \Sigma \Sigma p_{i} x_{i j} d_{i j}+p^{-}=P
\end{aligned}
$$

For performance based budgeting, the constraints are of the following types:

- The funds allocated to all programmes funded over a period to be equal to the available amount.

- The general funds allocated per programme, in the entire planning horizon, not to exceed the requested amount.

- The amounts allocated to each programme to be in the upper and lower thresholds for each period.

- The fundability of a programme in a period of the planning horizon. ie,

$$
\begin{aligned}
& \Sigma \mathrm{x}_{\mathrm{ij}}=\mathrm{f}_{\mathrm{j}} \\
& \Sigma \mathrm{x}_{\mathrm{ij}}=\mathrm{t}_{\mathrm{i}}
\end{aligned}
$$

Thus, we have the objectives and constraints based on Goal Programming(GP) which can be implemented in excel spreadsheet. Now we need to find the risk and priority input parameter values in the mathematical model based on GP using fuzzy logic which is described in the next section

\subsection{Fuzzy Tool for Quantification of Risk and}

\section{Priority}

The inputs to the goal programming model, risk and priority are quantified using fuzzy logic after mathematical modelling. Among the various quantification techniques available, fuzzy logic seems to be most appropriate as it enables the translation of linguistic variables to measurable quantities. It is difficult to quantify parameters like risk but can be well described in linguistic variables. So here fuzzy tool kit in matlab is used to quantify input parameters risk and priority. First of all a matrix is formulated as shown in fig-3.The bases of this matrix is that risk is a combination of severity and likelihood. Then the input and output membership functions are defined in the fuzzy toolkit. Here the input functions are severity and likelihood and risk is the output function. The Gaussian membership function is mostly accepted for risk type functions, hence employed here. Then the likelihood and severity of risk of each Technology Development Programme is compared with the risk assessment matrix shown in Table 2.

Table - 2: Risk Assessment Matrix

\begin{tabular}{|l|l|l|l|l|}
\hline \multicolumn{4}{|l|}{ Severity } \\
\hline Likelihood & Catastrophic & Critical & Marginal & Negligible \\
\hline Frequent & High & High & Serious & Medium \\
\hline Probable & High & High & Serious & Medium \\
\hline Occasional & High & Serious & Medium & Low \\
\hline Remote & Serious & Medium & Medium & Low \\
\hline Improbable & Medium & Medium & Medium & Low \\
\hline
\end{tabular}

For the purpose of this work, equally distributed ranges have been assigned to each risk level as shown in Table 3.

Table - 3: Case study risk levels

\begin{tabular}{|l|l|l|}
\hline & From & To \\
\hline Low & 0 & 0.25 \\
\hline Medium & 0.25 & 0.5 \\
\hline Serious & 0.5 & 0.75 \\
\hline High & 0.75 & 1 \\
\hline
\end{tabular}

Rule Viewer window in fig.4 shows the crisp or quantified value of risk for the sample input given as linguistic variables in Table 4. The sample input linguistic values based on expert opinion indicate that the programme considered here is having risk which is occasional and marginal in nature.

Table- 4: Test Input Sample

\begin{tabular}{|l|l|l|l|}
\hline & $\begin{array}{l}\text { Linguistic } \\
\text { value }\end{array}$ & $\begin{array}{l}\text { Calibrated } \\
\text { range }\end{array}$ & $\begin{array}{l}\text { Range } \\
\text { Test Value }\end{array}$ \\
\hline Likelihood & Occasional & $0.4-0.6$ & 0.5 \\
\hline Severity & Marginal & $0.25-0.5$ & 0.5 \\
\hline Risk (crisp output) & $\mathbf{0 . 5}$ \\
\hline
\end{tabular}

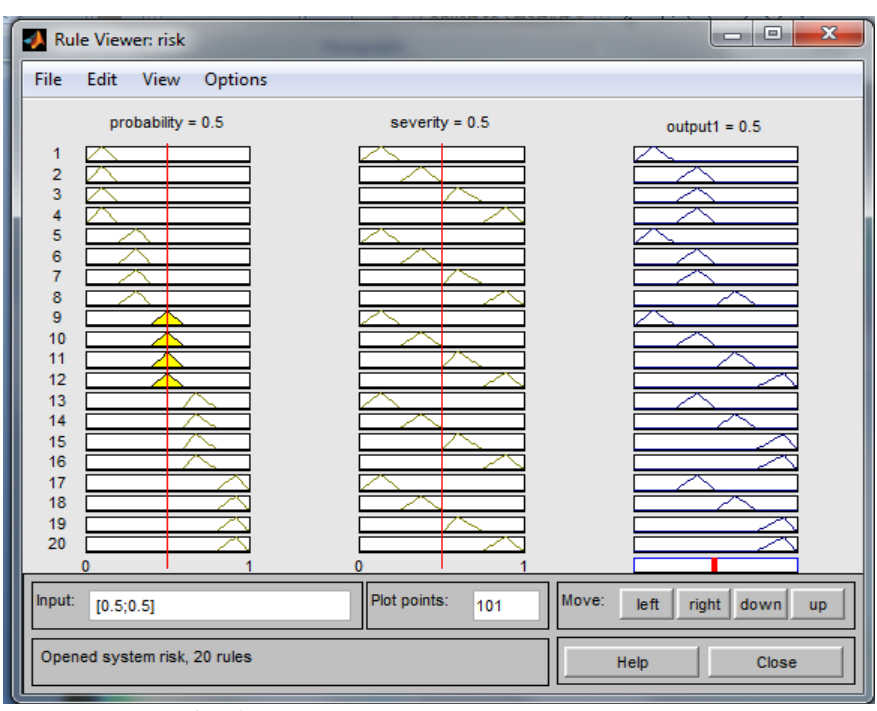

Fig-3: Rule Viewer crisp risk output 
As it is demonstrated in fig. 3, the generated output pattern tells that the risk of test input sample programme tends to be a medium risk level. Similarly for priority assessment the above procedures are repeated. Firstly a priority matrix is developed. Then membership functions are defined for input variable "Program Priority" and output variable "Expert Preference". Finally the priority of each Technology Development Programme is compared with the priority assessment matrix. The rule viewer gives the quantified values of priority for each programme.

\subsection{Spreadsheet Modeling and Excel Solver}

The mathematical model developed based on goal programming optimization method is exemplified here for a sample of five Technology Development Programmes(P1,P2,P3,P4 \&P5) from the concerned R\&D organization. The model is implemented in Microsoft Excel and optimized with its Solver optimization tool.

The Solver Parameters Dialog Box is used to implement mathematical model developed in excel spreadsheet. Clicking on Data $>$ Solver, the dialog box will open. We fill in the 'Set Objective' box by clicking on the cell in our spreadsheet that calculates our objective function. Next, we use the radio buttons below to identify the type of problem we are solving, a MAX or MIN. Then, we need to identify the decision variables. SOLVER terms these as variable cells. After clicking into the 'By Changing Variable Cells' box, we can select the decision variable cells in our problem. We need to add our constraints to SOLVER to ensure our solution that does not violate any of them. Additionally, we have to change the Select a Solving Method to SIMPLEX LP when we are solving a linear program. Finally, click Solve for getting the solution.

\section{CASE STUDY}

The performance-based Budgeting model described above with inputs quantified using fuzzy logic is exemplified here for a sample of five R\&D Programmes(P1,P2,P3,P4 \& P5) from an $R \& D$ organization. The risk and priority parameters quantified using fuzzy logic are given as inputs to mathematical model based on Goal Programming (GP) for determining the appropriate allocation of funds for R\&D programmes. The model is implemented in excel as shown in Fig-4 using its solver tool. The solver results show an improved allocation of funds over the existing thumb rule allocation. The results also imply that weights to decision variables can be changed according to the changing needs of the organization. The Experts of the programme can make suitable weight assignments to the decision variables. Also it is possible to analyze the impact of assigning different weights to the decision variables on the final objective function and allocated funds to different programmes by checking each scenario in solver with the advantage of time.

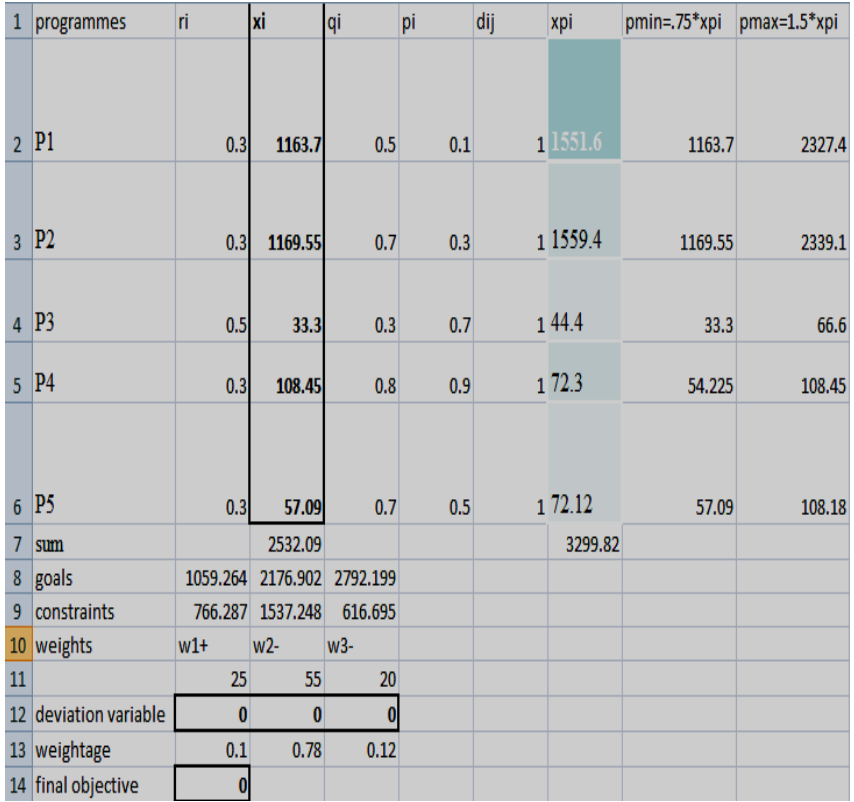

Fig-4: Excel Solver results of goal programming model

The allocated funds as per Goal Programming model is compared with the actual expenditure of the projects for the year 2013 as shown in Table 5. It is found that the actual expenditure values are closer to allocated values using the GP model rather than the actual allocation as per existing thumb rule. Hence we can say that the budget allocation gets improved using the performance based budgeting model. Once completed, the goal programming model built using a spreadsheet tool can be used as a DSS (Decision Support System) for the performance-based allocation of public funds.

Table- 5: Validation Results of the GP model

\begin{tabular}{|c|c|c|c|}
\hline Projects & $\begin{array}{c}\text { Actual } \\
\text { allocation in } \\
\mathbf{2 0 1 3} \text { as per } \\
\text { existing rule }\end{array}$ & $\begin{array}{c}\text { Actual } \\
\text { expenditure } \\
\text { data in } \\
\mathbf{2 0 1 3}\end{array}$ & $\begin{array}{c}\text { GP model } \\
\text { values using } \\
\text { fuzzy }\end{array}$ \\
\hline P1 & 1551.6 & 1048 & 1163.7 \\
\hline P2 & 1559.4 & 760 & 1169.55 \\
\hline P3 & 44.4 & 28 & 33.3 \\
\hline P4 & 72.3 & 92 & 108.45 \\
\hline P5 & 72.12 & 56.21 & 57.09 \\
\hline Total & $\mathbf{3 2 9 9 . 8 2}$ & $\mathbf{1 9 8 4 . 2 1}$ & $\mathbf{2 5 3 2 . 0 9}$ \\
\hline
\end{tabular}

The model values using fuzzy shows an overall improved allocation of $30.32 \%$ compared to thumb rule allocation. The improvement in allocation with GP model using fuzzy inputs for projects P1, P2, P3, P4, P5 are 33.33\%, 33.33\%, $33.33 \%, 33.33 \%$ and $26.33 \%$ respectively. Thus it is seen that the model values using fuzzy are more close to actual expenditure values compared to thumb rule allocation. Hence it is stated that the Goal Programming model with fuzzy gives a better allocation of funds than the existing system. The correlation coefficient and regression coefficient of model values and actual expenditure are calculated and the values obtained were 0.9759 and 0.9366 respectively which indicates that the model is effective. 


\section{CONCLUSION}

The study analyzed the present fund allocation and utilization in $R \& D$ over the last nine years. It was found that there exists a wide gap between the allocation and utilization of funds. A mathematical model based on goal programming technique was developed for performance based budgeting. The performance based budgeting model based on goal programming technique was found to be useful in optimizing the public funds allocation in a dynamic and complex environment. The work in this paper showed Fuzzy logic as an appropriate tool for the risk and priority quantification process. The quantified parameter values of risk and priority using fuzzy logic were used as inputs to the goal programming model. The goal programming model built using a spreadsheet tool was used as a DSS (Decision Support System) for the performance-based allocation of public funds. Several scenarios could be constructed using this model to support fact based decisions. The future study may incorporate more complex processes of $\mathrm{PB}$ as the present study considered only the final allocation of funds.

\section{REFERENCES}

[1]. Luminita Zamfirescu, Constantin-Bala Zamfirescu(2013)," Goal programming as a decision model for performance-based Budgeting", Procedia Computer Science, vol.17,pp $426-433$

[2]. Tamiz M, Jones D (1998) Goal programming: recent developments in theory and practice. Int $\mathrm{J}$

Manag Syst 14:1-16

[3]. Reina L (2005) From subjective expected utility theory to bounded rationality: an experimental

investigation on categorization processes in integrative negotiation, in committees' decision

making and in decisions under risk. Doctorate thesis, Technische Universit"at Dresden

[4]. Romero C (1991) Handbook of critical issues in goal programming. Pergamon, Oxford

[5]. Romero C (2004) A general structure of achievement function for a goal programming model. Eur J Oper Res 153:675-686

[6]. Chang C-T (2007) Efficient structures of achievement functions for goal programming models.Asia Pac J Oper Res 24(6):755-764

[7]. Charnes A, Cooper W, Ferguson R (1955)," Optimal estimation of executive compensation by linear programming", Journal of Management science,pp 138-151 [8]. Tamiz M, Jones D (1995)," A review of goal programming and its applications", Ann Oper Res, pp 39-53 [9]. Chen, S.J. and Chen, S.M., "Fuzzy risk analysis based on similarity measures of generalized fuzzy numbers," IEEE Transactions and Fuzzy Systems, vol. 11, pp. 45- 56, 2008. [10]. P. Rezakhani,(2012)," A review of fuzzy risk assessment models for construction projects",Slovak Journal of Civil Engineering, Vol. XX, 2012, No. 3, 35 - 40

[11]. Zeng, J., An, M., and Smith, N. J. (2007). Application of a fuzzy based decision making methodology to construction project risk assessment. International Journal of Project Management, 25, 589-600.
[12]. Azmi R and Tamiz M,(2010)," A Review of Goal Programming for Portfolio Selection", New Developments in Multiple Objective and Goal Programming,vol.638 